\title{
Auxiliary partial orthotopic liver transplantation in pre-eclampsia
}

\author{
Sanggyun Suh, Kyung-Suk Suh, Kwang-Woong Lee, Nam-Joon Yi, YounRok Choi, Suk Kyun Hong, Jeong-Moo Lee, \\ Kwangpyo Hong, Eui Soo Han
}

Division of Hepatobiliary, Department of Surgery, Seoul National University Hospital, Seoul, Korea

Background: Auxiliary partial orthotopic liver transplantation (APOLT) is a technically challenging procedure in which a segmental liver graft is implanted orthotopically following a native liver partial hepatectomy. Despite being considered an innovative technique, it is gradually gaining acceptance as a bridge therapy in acute liver failure with the possibility of immunosuppression free survival. Herein, we report a 35 -year-old pregnant woman with HELLP syndrome who underwent APOLT.

Methods: She was diagnosed with HELLP syndrome because of hemolysis (hemoglobin, $6.3 \mathrm{~g} / \mathrm{dL}$ ), elevated liver enzyme (aspartate aminotransferase/alanine aminotransferase, $11,783 / 8,288 \mathrm{IU} / \mathrm{L})$, and low platelet count $\left(56 \times 10^{3} / \mu \mathrm{L}\right.$ ) without evidence of other causes of hepatitis. She developed hepatic encephalopathy and eventually required ventilator and renal replacement therapy. After emergent C-section, she referred to acute to our center for management of fulminant liver failure (United Network for Organ Sharing status 1).

Results: She underwent APOLT from a 35-year-old deceased donor on hospital day 9. After right hemihepatectomy of the recipient, a right trisection segment graft was transplanted orthotopically. Cold ischemic time was not recorded and warm ischemic time was 33 minutes. Operation time of the recipient was 530 minutes and estimated blood loss was 5,000 mL. She was discharged on postoperative day 51. Initially she took triple therapy of immunosuppressants (tacrolimus, steroid, and mycophenolated mofetil) with Simulect. Her immunosuppressants were tapered down to tacrolimus monotherapy. Three-year follow-up biopsy of both livers showed acute cellular rejection of the graft and native liver well functioned. Thus, it was not treated. On a 5 -year follow-up computed tomography scan, the graft liver showed atrophy. She is free from immunosuppressants without complication related to graft and native liver on a 7-year follow-up.

Conclusions: APOLT is technically challenging procedure, but there is chance of immunosuppression-free survival. So we have to consider about APOLT as option for acute liver failure patient.

Corresponding author: Nam-Joon Yi

E-mail: gsleenj@hanmail.net

\section{(c) The Korean Society for Transplantation}

This is an Open Access article distributed under the terms of the Creative Commons Attribution Non-Commercial License (http://creativecommons.org/licenses/by-nc/4.0/) which permits unrestricted non-commercial use, distribution, and reproduction in any medium, provided the original work is properly cited. 